\title{
3000 In-Stent Restenosis of Carotid and Technical Vertebral Arteries Treated by Angioplasty Using a Cutting Balloon: A Case Report
}

Rin Shimozato, ${ }^{1}$ Yukihiro Hidaka, ${ }^{1}$ Daichi Nakagawa, ${ }^{2}$ Mototaka Hayashi, ${ }^{3}$ Yoshie Fuseya, ${ }^{4}$ and Akira lijima ${ }^{1}$

Objective: We treated a patient with internal carotid artery and vertebral artery ostium in-stent restenosis (ISR) treated by cutting balloon (CB) angioplasty.

Case Presentation: A 79-year-old man developed dizziness and right homonymous upper quadrantanopia. On arrival, magnetic resonance imaging (MRI) revealed acute-stage brain infarction. Angiography demonstrated left internal carotid artery and vertebral artery ostium stenosis (VAOS), which was thought to be related to the infarction. We performed stenting for both lesions, but 5 months later, restenosis occurred. The patient was successfully retreated by $\mathrm{CB}$ angioplasty for both lesions.

Conclusion: When treating carotid or vertebral artery ISR, plain balloon (PB) and stent-in-stent (SIS) procedures may induce insufficient dilatation, and hamper re-retreatment because of neointimal hyperplasia. Using CB should be considered as an option in such cases.

Keywords > in stent restenosis, cutting balloon, percutaneous transluminal angioplasty, vertebral artery, endovascular

\section{Introduction}

Percutaneous transluminal angioplasty (PTA) is a treatment option for internal carotid artery stenosis (ICAS) or vertebral artery ostium stenosis (VAOS) resistant to medical treatment. However, this procedure with a balloon alone results in insufficient dilatation or restenosis in many cases; therefore, stenting is often selected. However, the incidence of in-stent restenosis (ISR) remains high, especially in patients with VAOS. ${ }^{1-3)}$

${ }^{1}$ Department of Neuroendovascular Therapy, JCHO Tokyo Shinjuku Medical Center, Tokyo, Japan

${ }^{2}$ Department of Neurosurgery, JCHO Tokyo Shinjuku Medical Center, Tokyo, Japan

${ }^{3}$ Department of Neuroendovascular Therapy, Mito Brain Heart Center, Mito, Ibaraki, Japan

${ }^{4}$ Physiological Laboratory, JCHO Tokyo Shinjuku Medical Center, Tokyo, Japan

Received: June 9, 2020; Accepted: December 13, 2020 Corresponding author: Rin Shimozato. Department of Neuroendovascular Treatment, JCHO Tokyo Shinjuku Medical Center, 5-1, Tukudocho, Shinjuku-ku, Tokyo 162-8543, Japan Email:imnrstz@gmail.com

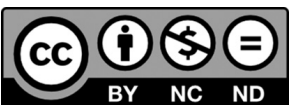

This work is licensed under a Creative Commons Attribution-NonCommercialNoDerivatives International License.

(C)2021 The Japanese Society for Neuroendovascular Therapy
On the other hand, few studies have reported additional treatment for ISR in patients with VAOS.

In this study, we report a patient in whom carotid artery stenting for ICAS and vertebral artery stenting for VAOS were performed, and PTA with a cutting balloon (CB) for ISR of the two arteries led to a favorable course.

\section{Case Presentation}

Patient: A 79-year-old man.

Medical history: Hyperlipidemia.

Lifestyle: Occasional alcohol consumption and smoking (5-10 cigarettes/day).

Present illness: At night, dizziness, dysarthria, and weakness of the right half body were noted, but he did not call an ambulance. In the morning (after 12 hours), vomiting developed and he was brought to our hospital by ambulance.

Physical findings: The blood pressure, oxygen saturation of the peripheral artery $\left(\mathrm{SpO}_{2}\right)$, and pulse rate were 151/75 $\mathrm{mmHg}, 97 \%$ (room air), and 82 beats/min, respectively. Electrocardiography demonstrated sinus rhythm. His consciousness was clear. There was no limb paralysis. The National Institutes of Health Stroke Scale (NIHSS) score was 1 ("ataxia" section).

Imaging findings: On magnetic resonance imaging (MRI)diffusion-weighted imaging (DWI), high signal intensity 


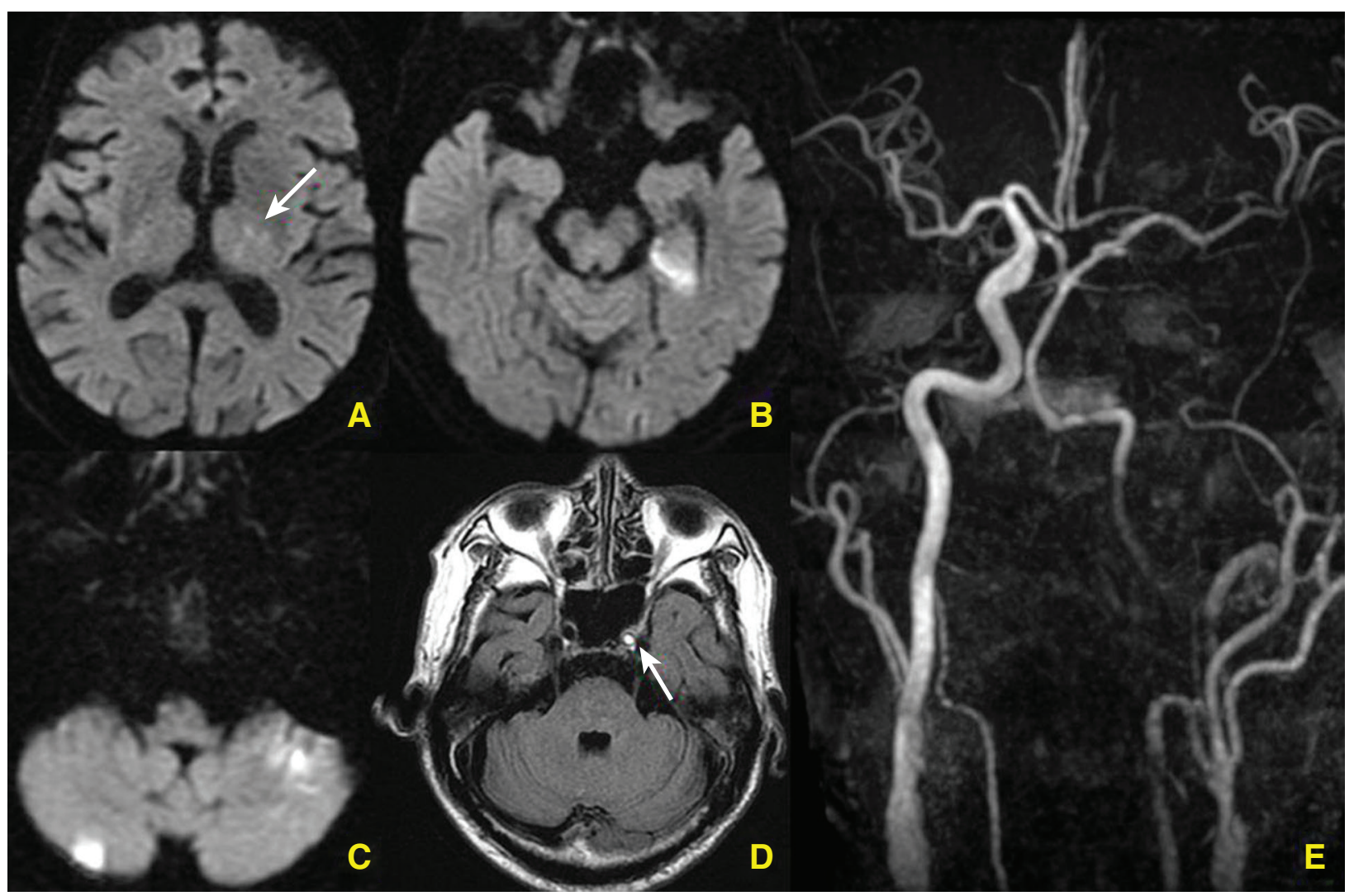

Fig. 1 (A-C) Diffusion-weighted MRI shows high-intensity lesions in the left internal capsule (white arrow), hippocampus, and bilateral cerebellar hemispheres. (D) MRI FLAIR image shows intra-arterial sign in the left internal carotid artery (white arrow). (E) MRA of bilateral carotid to cerebral arteries shows obliteration of the left internal carotid artery, and flow in the left middle cerebral artery via the anterior communicating artery and posterior communicating artery. involving the posterior limb of the left internal capsule, medial left temporal lobe, and bilateral cerebellar hemispheres was observed. Magnetic resonance angiography (MRA) revealed occlusion of the left internal carotid and right vertebral arteries. Left middle cerebral artery blood flow mediated by the anterior and left posterior communicating arteries was suggested (Fig. 1).

Course after admission: The patient was admitted to the Department of Internal Medicine. The intravenous injection of heparin sodium at 10000 units/day and oral administration of clopidogrel at $75 \mathrm{mg}$ were started. On MRI on Day 6, scattered infarction of the left frontal lobe and infarction of the left occipital lobe were observed. MRA confirmed recanalization of the left internal carotid artery (Fig. 2). Right upper quadrantanopia was noted and the patient was referred to our department.

Cerebral blood flow scintigraphy was performed on Day 15 and cerebral angiography on Day 17.

On resting blood flow scintigraphy with IMP, there was no laterality. Cerebral angiography revealed left ICAS (distal vascular diameter: $5.3 \mathrm{~mm}$, vascular diameter at the stenotic site: $1.2 \mathrm{~mm}$, percent stenosis: 77\%, North American Symptomatic Carotid Endarterectomy Trial; NASCET, Criteria) and left VAOS (distal vascular diameter: $3.5 \mathrm{~mm}$, vascular diameter at the stenotic site: $0.8 \mathrm{~mm}$, percent stenosis: $77 \%$ as a percentage of the distal vascular diameter at the non-stenotic site). Anastomosis formation from the left occipital artery to the left vertebral artery was found, reflecting a decrease in the volume of blood flow to the vertebral artery. Hypoplasia of the right vertebral artery was noted (Fig. 3). Ultrasonography demonstrated low-echoic plaque of the internal carotid artery, a peak systolic velocity (PSV) of $300 \mathrm{~cm} / \mathrm{s}$, low- to iso-echoic plaque of the vertebral artery, and a PSV of $30.5 \mathrm{~cm} / \mathrm{s}$.

We considered arteriogenic embolism rather than hemodynamic infarction for the following reasons: there was no laterality on blood flow scintigraphy and infarction was not typical at the watershed area. Although infarction involved the supra- and infratentorial areas, thrombi at the origin of the internal carotid artery before recanalization may have been scattered from the occipital artery to the vertebral artery via the external carotid artery, inducing anterior/ 


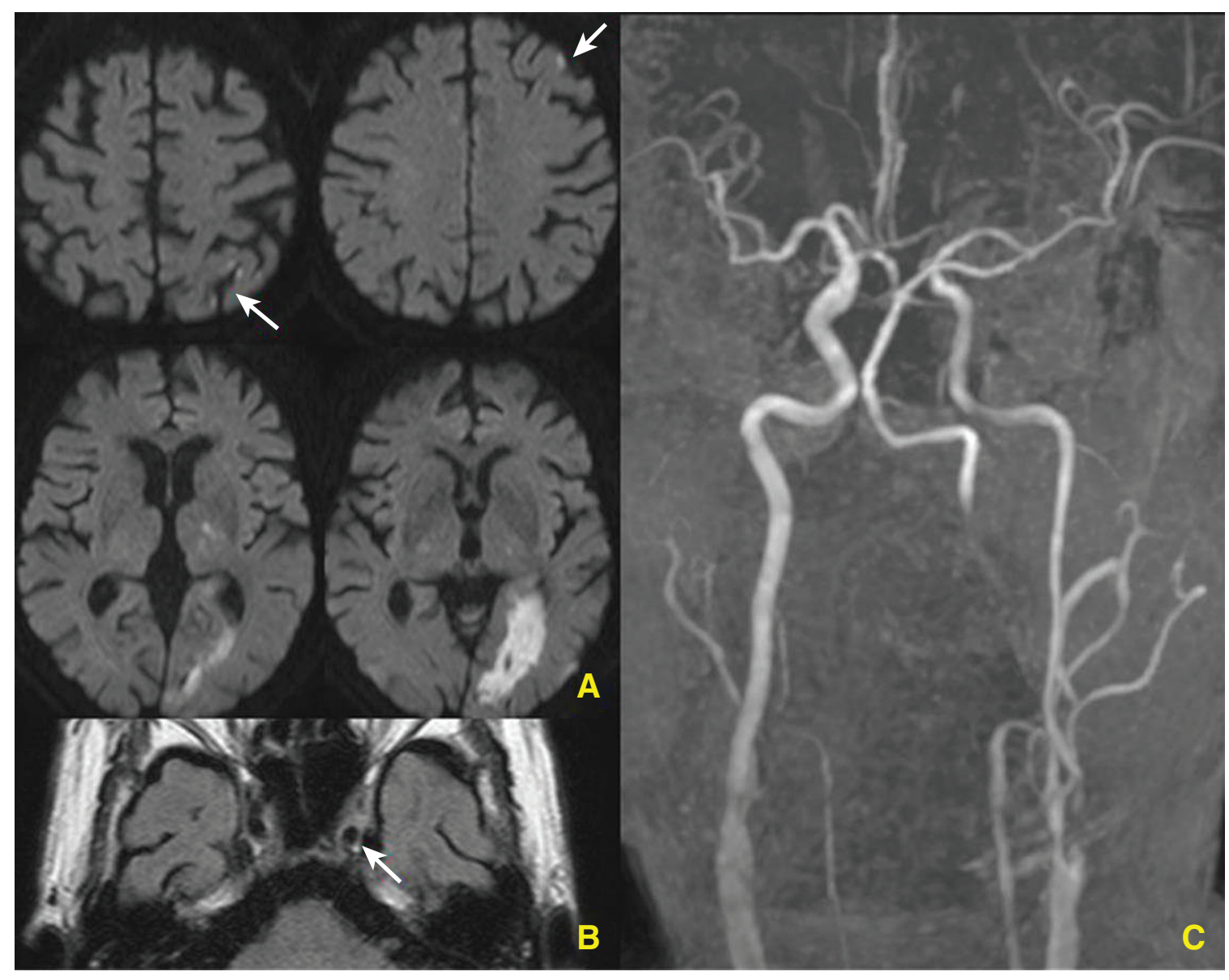

Fig. 2 (A) Diffusion-weighted MRI lesions in the left frontal lobe (white arrows) and left occipital lobe. (B) MRI FLAIR image showing the disappearance of the intra-arterial sign in the left internal carotid artery (white arrow). (C) MRA of bilateral carotid to cerebral arteries shows recanalization in the left internal carotid artery.

posterior circulation infarction considering the presence of occipital-to-vertebral artery anastomosis and occlusion of the internal carotid artery before recanalization.

To prevent recurrent infarction, revascularization of the two lesions was scheduled. The internal carotid artery was considered to be a direct responsible lesion, but we adopted a strategy to initially perform angioplasty of the vertebral artery and treat the left internal carotid artery after reducing anastomotic-pathway-mediated blood flow considering the risk of embolic complications at this site related to an anastomotic pathway to the vertebral artery by the occipital artery. Dual antiplatelet therapy (DAPT) with aspirin at $100 \mathrm{mg} /$ day and clopidogrel at $75 \mathrm{mg} /$ day was performed. Under local anesthesia, a 7Fr angle-type guiding catheter (ASAHI FUBUKI Angled: ASAHI INTECC, Aichi, Japan) was inserted into the left subclavian artery through the right femoral artery for systemic heparinization. Under distal protection of the left vertebral artery with a balloon system (PercuSurge; Medtronic, Minneapolis, MN, USA), dilatation with a balloon measuring $2.5 \times 10 \mathrm{~mm}$ (Unryu; Kaneka Medics, Kanagawa, Japan) was performed, but dilatation was insufficient. A $3.5 \times 23-\mathrm{mm}$ coronary stent (Multi link; Abbott Vascular, Santa Clara, CA, USA) was coaxially inserted. Aspiration was performed using an aspiration catheter to deflate the PSGW. After treatment, favorable dilatation similar to that in the normal area was achieved at the stenotic site (Fig. 4). With a favorable increase in the volume of vertebral artery/intracranial blood flow distal to the stenotic site, the volume of blood flow related to a collateral pathway from the occipital artery decreased, but this did not lead to complete disappearance. After 8 days, angioplasty of the carotid artery was performed. As described for initial angioplasty, systemic heparinization was conducted under local anesthesia. A 9Fr guiding catheter (Optimo; Tokai Medical Products, Aichi, Japan) was inserted into the left common carotid artery through the left femoral artery. As the anastomotic pathway from the occipital artery to the vertebral artery remained, a Carotid Wall 


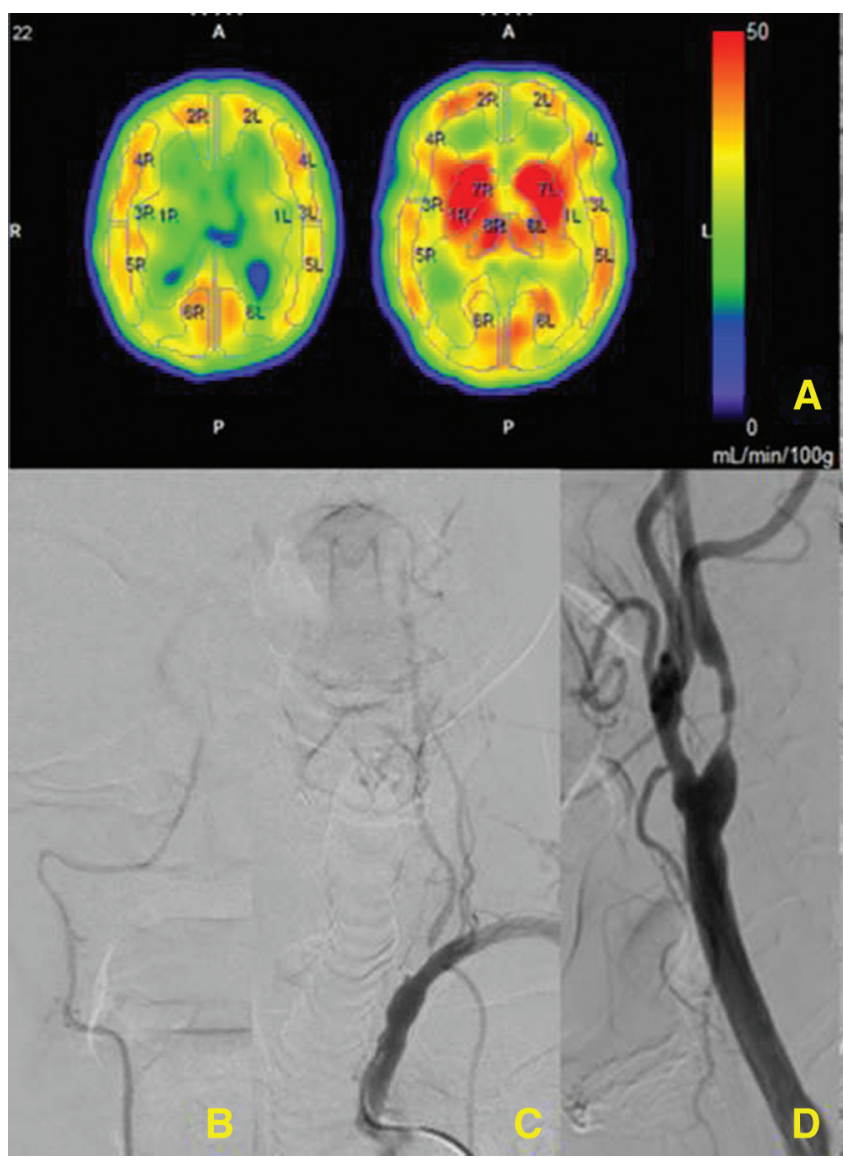

Fig. 3 (A) IMP-SPECT imaging shows no cerebral blood flow (mL/ $\mathrm{min} / 100 \mathrm{~g}$ ) laterality. (B and C) Subclavian angiography shows right vertebral artery hypoplasia and left vertebral artery origin stenosis. (D and E) Left common carotid angiography shows ICAS and collateral

Stent $10 \times 31 \mathrm{~mm}$ (Stryker, Kalamazoo, MI, USA) was coaxially deployed after predilatation with a $3.5 \times 20-\mathrm{mm}$ balloon (Rx-Genity; Kaneka Medical Products, Tokyo, Japan) under protection of the external and internal carotid arteries with a PSGW, and postdilatation with a $5.0 \times 20-\mathrm{mm}$ balloon (Rx-Genity) was conducted (Fig. 4). Favorable dilatation was achieved and there was no perioperative complication. The patient was discharged with a modified Rankin Scale (mRS) score of 1. On ultrasonography, the PSV of the internal carotid artery was $68 \mathrm{~cm} / \mathrm{s}$ and that of the vertebral artery was $74 \mathrm{~cm} / \mathrm{s}$. After DAPT was continued for 3 months, it was switched to monotherapy with clopidogrel.

Restenosis of the internal carotid and vertebral arteries was observed 5 months after surgery (internal carotid artery: distal vascular diameter, $5.3 \mathrm{~mm}$; vascular diameter at the stenotic site, $1.4 \mathrm{~mm}$; percent stenosis, 74\%, NASCET Criteria; and vertebral artery: distal vascular diameter, $3.5 \mathrm{~mm}$; vascular diameter at the stenotic site, $1.4 \mathrm{~mm}$; percent stenosis, $60 \%$ as a percentage of the distal vascular diameter at the non-stenotic site). Ultrasonography demonstrated iso-echoic plaque of the

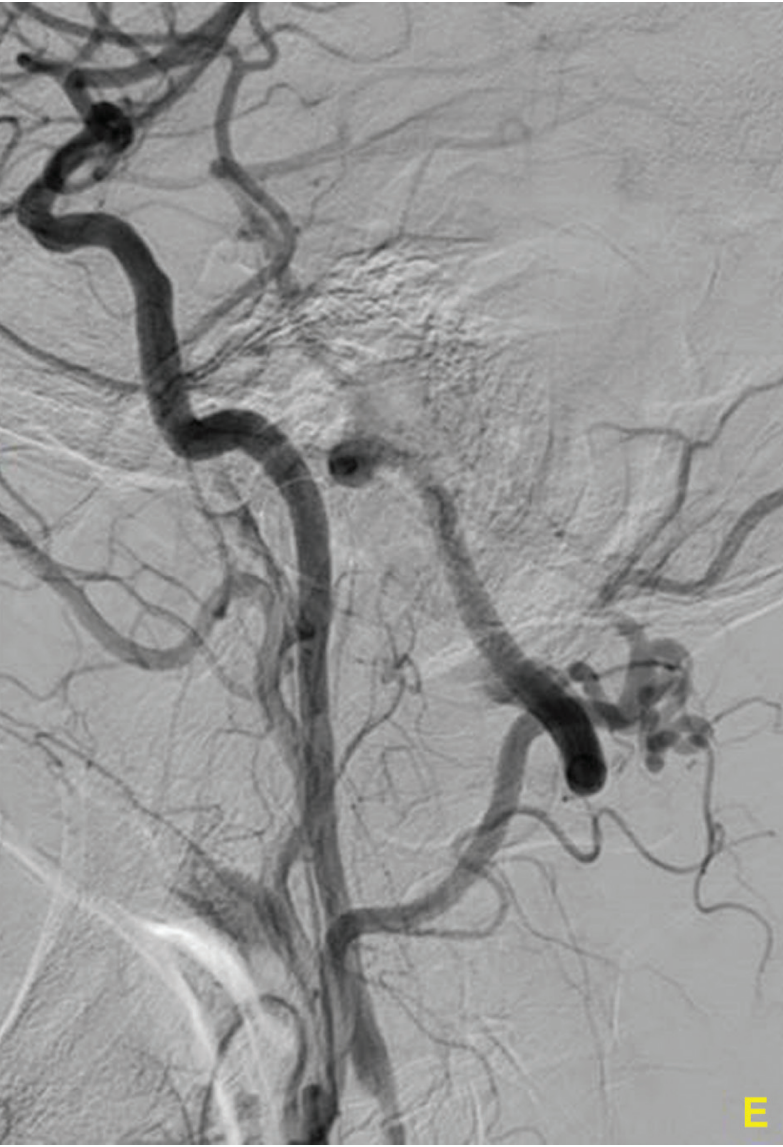

flow of the left vertebral artery through the left occipital artery. ICAS internal carotid artery stenosis; IMP-SPECT: 123-I-iodoamphetamine-single photon emission computed tomography

internal carotid artery and a PSV of $251 \mathrm{~cm} / \mathrm{s}$. It was difficult to evaluate the brightness of plaque of the vertebral artery and the PSV was $17.7 \mathrm{~cm} / \mathrm{s}$. To investigate the etiology, a platelet aggregation test was conducted. There was no suppression of adenosine 5'-diphosphate (ADP)-induced platelet aggregation, suggesting a poor response to clopidogrel. Based on the results of a patch test for investigating metallic allergy, chromium, nickel, or titanium allergy was excluded. As marked progressive restenosis was noted, additional treatment was planned.

The course suggested ISR in the chronic phase; at the two sites, fibrous stenosis with intimal hyperplasia may have been primarily involved in the pathogenesis. Furthermore, at the two sites, the etiology of intimal hyperplasia was unclear and we considered similar stenting to further induce intimal hyperplasia. Therefore, we did not select the stent-in-stent (SIS) procedure. Then, we devised angioplasty with a $\mathrm{CB}$ because resistance to treatment using a plain balloon (PB) was observed on initial treatment.

Endovascular treatment for ISR (in-stent PTA of the vertebral/carotid arteries with a $\mathrm{CB}$ ): The preoperative 


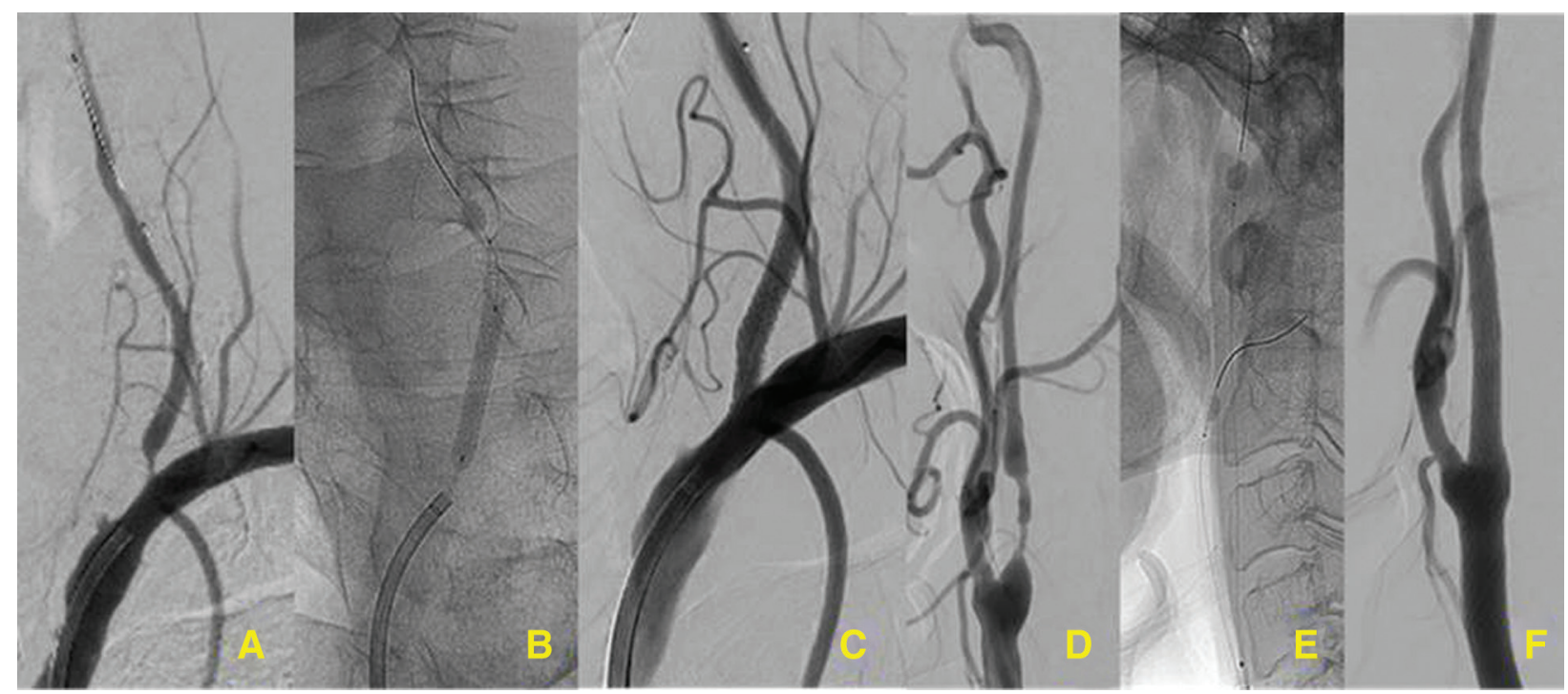

Fig. 4 (A-C) Left subclavian angiography shows vertebral artery dilatation by stenting. (D-F) Left common carotid angiography shows internal carotid artery dilatation by stenting. We used a dou- ble protection system by PSGWs to protect the internal carotid artery and vertebral artery, followed by the external carotid artery and occipital artery. medications were switched to a combination of aspirin at $100 \mathrm{mg} /$ day and cilostazol at $200 \mathrm{mg} /$ day. Using a system similar to that used in the previous session, a PSGW was inserted into the distal vertebral artery via the stent lumen and dilated. A Wolverine CB $3.0 \times 10 \mathrm{~mm}$ (Boston Scientific, Marlborough, MA, USA) was dilated at 8 atm (nominal: $6 \mathrm{~atm}$, rated burst pressure [RBP]: $12 \mathrm{~atm}$ ) at the site of in-stent stenosis and maintained for 30 seconds. After confirming the absence of changes in vitals, dilatation at 12 atm for 30 seconds was added.

After 3 months, carotid artery treatment using a $\mathrm{CB}$ was performed. An 8Fr ST-type guiding catheter (ASAHI FUBUKI Angled; ASAHI INTECC) was inserted into the left common carotid artery. As an area to be dilated was distal to the origin of the carotid artery, an $8 \mathrm{Fr}$ catheter was selected to protect the internal carotid artery alone. However, anastomosis from the occipital artery to the vertebral artery disappeared with an increase in the volume of vertebral artery blood flow, securing the safety. A Flextome Peripheral CB $4.0 \times 15 \mathrm{~mm}$ (Boston Scientific) was dilated to 8 atm (nominal: $6 \mathrm{~atm}, \mathrm{RBP}: 12 \mathrm{~atm}$ ) in the stent and maintained for 30 seconds. In addition, similar dilatation was added using the same balloon on the proximal side (Fig. 5).

\section{Postoperative course}

Favorable dilatation was achieved and there was no perioperative complication. The patient was discharged with a $\mathrm{mRS}$ score of 0 . DAPT is being continued. Ultrasonography 6 months after vertebral artery treatment and 3 months after internal carotid artery treatment demonstrated no changes in accelerated blood flow (PSV of the internal carotid artery: $133 \mathrm{~cm} / \mathrm{s}$, PSV of the vertebral artery: $86 \mathrm{~cm} / \mathrm{s}$ ) (Fig. 5).

\section{Discussion}

In patients with medical-treatment-resistant and acutely progressive ICAS or VAOS, PTA is selected as additional treatment. Stenting is well known to be effective for ICAS. Concerning VAOS, PTA-resistant factors include calcification and an elastic force specific to the origin of an artery.,5) PTA with a balloon alone results in insufficient dilatation or restenosis at a high incidence; therefore, this procedure is combined with stenting. However, ISR may occur with a probability of $21 \%-38 \% .^{1-3)}$ Plaque protrusion or platelet aggregation due to injury/dissection of the intima or a stent itself $^{6}$ is involved in the pathogenesis of acute-phase ISR. In-stent neointimal hyperplasia is involved in the pathogenesis of remote-phase ISR. Factors for the former include plaque properties, unresponsiveness to antiplatelet drugs, insufficient stent dilatation, and insertion to an inadequate position. As factors for the latter, stenting-related arterial injury and fibroproliferative responses related to delayed hypersensitivity in patients with metal allergy were reported. ${ }^{7,8)}$

In the present case, restenosis was noted 5 months after treatment. As there was no stenosis on any examination during admission, the latter factors were suspected, but platelet aggregation and metal patch tests were conducted 


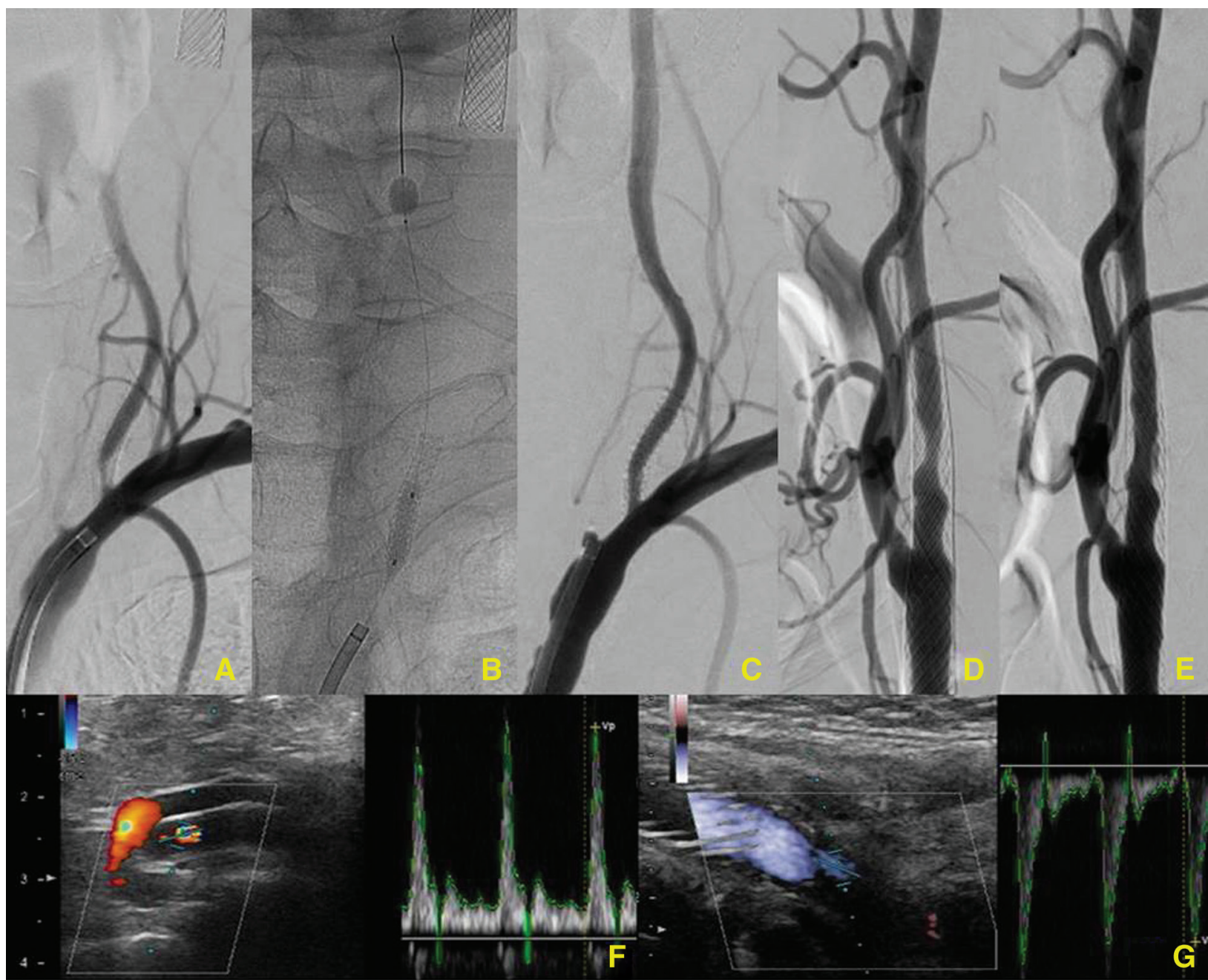

Fig. 5 (A-C) Left subclavian angiography shows vertebral artery origin ISR and dilatation by CB angioplasty. ( $\mathbf{D}$ and $\mathbf{E}$ ) Left common carotid angiography shows internal carotid artery ISR and dilatation by $\mathrm{CB}$ angioplasty. ( $\mathbf{F}$ and $\mathbf{G}$ ) The ultrasound examination performed after 3 months showed good blood flow. In this case, the normal systolic velocity of the vertebral artery indicated vascular patency. CB: cutting balloon; ISR: in-stent restenosis to investigate the etiology. As a result, the platelet aggregation test suggested a poor response to clopidogrel and the antiplatelet drug was switched.

Therapeutic strategies include in-stent PTA and SIS. For PTA, a PB, CB, or drug-coated balloon (DCB) is selected as an option. For stenting, a bare metal stent (BMS) or drug-eluting stent (DES) is selected. Internationally, several studies reported the use of a DCB or DES in the head and neck, but this is avoided in Japan considering the neurotoxicity of the coating drug.

The SIS procedure has demerits: the risk of intimal hyperplasia related to overlapped stents and in-stent delivery.

CBs are primarily used in the coronary artery and peripheral limb areas. On the balloon surface, a metallic microblade is longitudinally attached. When a balloon is dilated, the blade folded in the balloon appears on the surface, being pushed to plaque and facilitating cutting; a target site can be dilated at a low pressure (Fig. 6A). Several studies reported that $\mathrm{CBs}$ were effective for restenosis after carotid artery stenting. ${ }^{9-11)}$

Concerning the mechanism, a microblade on the $\mathrm{CB}$ surface may reduce recoil tension ${ }^{9)}$ through neointimal cutting, ${ }^{9,10,12)}$ pushing fragmented intimal tissues out of a stent. ${ }^{10,12)}$ Luminal gain may be achieved without inducing vascular-wall or stent overexpansion, that is, without increasing the outer diameter of a blood vessel, ${ }^{9,12,13)}$ differing from conventional balloons. A prospective randomized study regarding ISR in the coronary region demonstrated that slippage episodes were significantly less frequent. ${ }^{14)}$ As a CB-related risk, microblade-related vessel injury may occur, but an initially inserted stent functions as a barrier in the case of ISR; therefore, dilatation up to the diameter of 


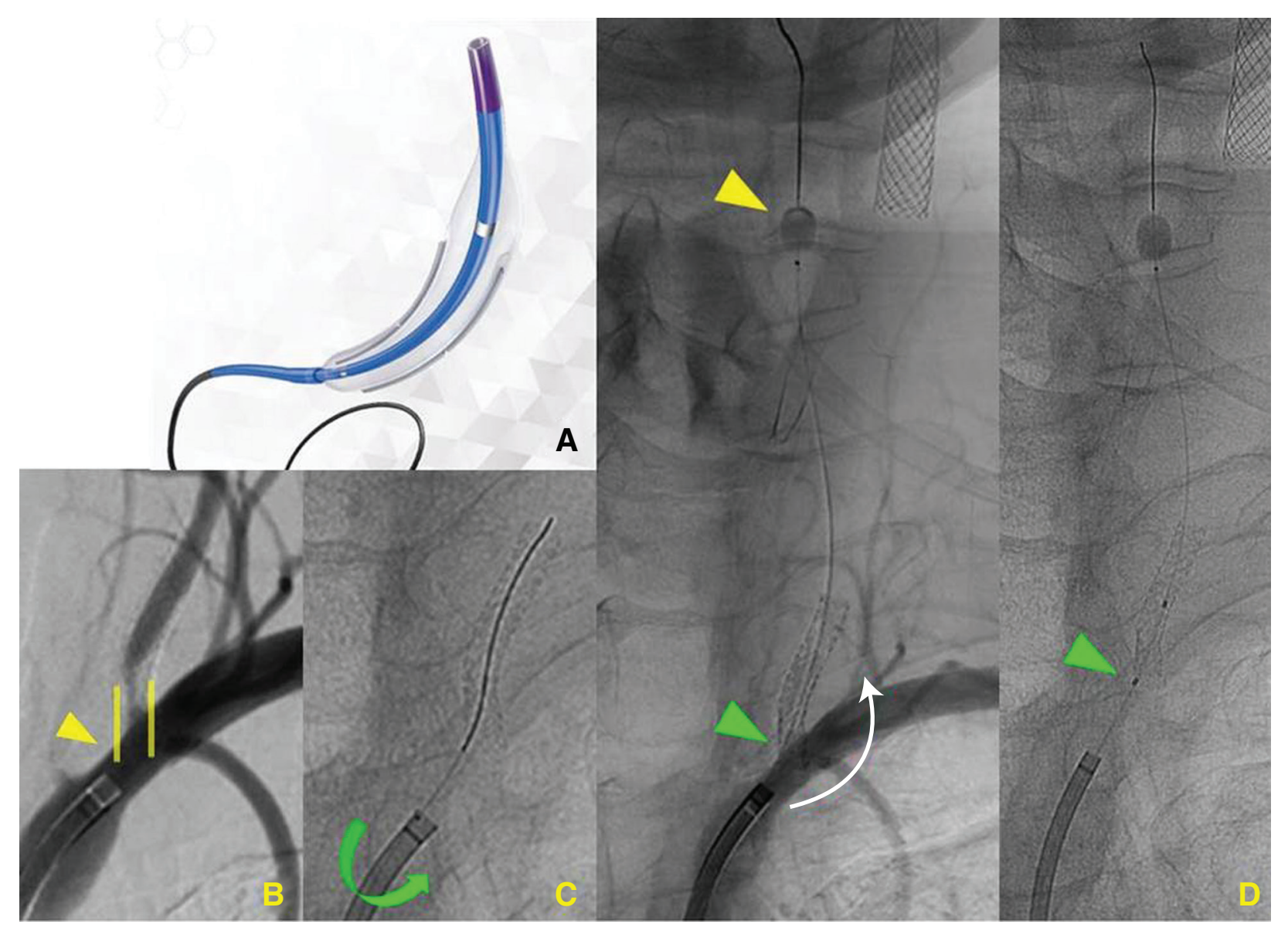

Fig. 6 (A) Schema of the CB: A few microblades made of stainless steel slice the intimal tissue (Wolverine cutting balloon, Boston Scientific). (B-D) Left subclavian angiography. (B) The stent edge was sticking out into the subclavian artery (parallel yellow lines and yellow arrowhead). (C) The PSGW was prevented from becoming stuck in the stent by rotating the guiding catheter (curved green arrow). (D) We bent the PSGW by pushing it after the inflated balloon of the tip anchors (curved white arrow, yellow arrowhead). As such, the mounting angioplasty balloon (in this case, CB) had no interference in the stent (green arrowheads). CB: cutting balloon the initially inserted stent may be safe. ${ }^{10)}$ Furthermore, intimal overreactions to a foreign body (i.e., another stent) can be avoided, which is an advantage. ${ }^{11}$ )

Previous studies reported that the use of a CB in carotid artery stenting was highly safe, but a study regarding its use in the coronary region found that the incidence of myocardial infarction within 30 days was high; therefore, caution is needed. The denuded re-endothelialized stent struts as a factor for thrombus formation may induce platelet activity. The above study recommended postoperative combination therapy with two antiplatelet drugs. ${ }^{15)}$ In addition, the use of this balloon for head and neck lesions is not covered by health insurance. Therefore, this balloon should be used only in the case that there has been no evidence on the current treatment methods or in conventional treatment-resistant patients.

In Japan, the SIS procedure is frequently selected to treat ISR after stenting for ICAS. In our department, it is also selected as a first-choice procedure for acute-phase
ISR related to soft plaque such as plaque protrusion. If possible, atraumatic pre-/postdilatation-free stenting should be performed to correct/stabilize the base with an irregular wall. On the other hand, chronic-phase ISR may consist of fiber components, and PTA is selected considering that dilatation may not be achieved using the standard SIS procedure alone and that intimal hyperplasia may further occur. In this case, as a rule, PTA with a PB is adopted as a firstchoice procedure, but the use of a $\mathrm{CB}$ is considered when treatment resistance is noted.

For the vertebral artery, as a rule, PTA with a PB is selected for acute-phase ISR, and this procedure is initially selected for chronic-phase ISR consisting of fiber components for the following reasons: intimal hyperplasia may make treatment more difficult when placing two stents because the vascular diameter is smaller than that of the carotid artery, and device access is relatively difficult because of the same reason. 
In the present case, restenosis developed at two points in a short period, suggesting the risk of recurrence. Furthermore, the lesions were resistant to PBs on initial treatment, suggesting a fibrous factor. Therefore, we attempted in-stent PTA with a CB for VAOS in addition to ICAS, expecting effects similar to those in the carotid artery. To our knowledge, no study has reported the use of a CB for VAOS.

To treat ISR due to VAOS, the SIS procedure was adopted in previously reported patients. A comparative study with balloon angioplasty recommended the addition of a stent rather than treatment using a balloon alone as a first option. ${ }^{16}$ ) But this study was comparative with a PB. Furthermore, the long-term outcome is unclear and the possibility of further intimal hyperplasia cannot be excluded. If the SIS procedure is performed once, correction, that is, retrieval, may be impossible. Therefore, we consider it a final strategy.

The technical limitation of treatment for vertebral artery ISR is the difficulty of device delivery via the site of in-stent stenosis. A stent at the origin of the vertebral artery must be placed such that the entire length of stenosis at its origin is covered; therefore, the stent edge partially protrudes to the subclavian artery side in many cases. Indeed, the balloon part of the PSGW interfered with the stent edge on the subclavian artery side. We used an angled guiding catheter and interference at the balloon part of the PSGW was relieved by transmitting/rotating torque to the guiding catheter in the subclavian artery into which the guiding catheter was inserted, facilitating access of the PSGW into the stent. Subsequently, an interfering motion at the stent edge was also observed when guiding a $\mathrm{CB}$, but the interference was removed by rotating the guiding catheter, as described above. Furthermore, wire deflection at the stent orifice obtained by lightly pushing the PSGW wire in hand utilizing anchoring of the PSGW, which was dilated at a distal area, was useful for smoothly guiding the CB into the stent. Such an operation cannot be conducted using a filter-type protection device with which distal anchoring is not achieved, and the delivery of a collecting sheath is required for a filter-type device when collecting the device after the procedure. Thus, considering these points, a balloon-type device should be used (Fig. 6B-6D).

In the present case, the blood flow velocity in the vertebral artery before dilatation was low but increased after dilatation, exhibiting a paradoxical phenomenon. As a reason why the PSV of the vertebral artery before dilatation was low, we think as follows. A collateral pathway from the occipital artery was a primary blood flow tract and a low velocity was possibly measured through an interference with blood flow from the occipital artery despite the presence of post-stenotic affected-side vertebral artery anterograde blood flow, differing from accelerated blood flow, which is commonly observed in the presence of stenosis. After dilatation, the velocity of essential anterograde blood flow from the origin of the vertebral artery may have increased following attenuation of collateral pathway blood flow.

The long-term results should be examined in the future. However, the use of a CB may be considered as an option of stent-free additional treatment for vertebral artery ISR.

\section{Conclusion}

We report a patient in whom the use of a CB for ISR due to stenotic lesions of the carotid/vertebral arteries led to favorable dilatation.

This method may be an option for refractory ICAS and VAOS.

\section{Disclosure Statement}

The authors declare no conflicts of interest.

\section{References}

1) Ogilvy CS, Yang X, Natarajan SK, et al: Restenosis rates following vertebral artery origin stenting: does stent type make a difference? J Invasive Cardiol 2010; 22: 119-124.

2) Langwieser N, Prothmann S, Buyer D, et al: Safety and efficacy of different stent types for the endovascular therapy of extracranial vertebral artery disease. Clin Res Cardiol 2014; 103: 353-362.

3) Sun X, Ma N, Wang B, et al: The long term results of vertebral artery ostium stenting in a single center. J Neurointerv Surg 2015; 7: 888-891.

4) Hatano $T$, Tsukahara $T$, Miyakoshi A, et al: Stent placement for atherosclerotic stenosis of the vertebral artery ostium: angiographic and clinical outcomes in 117 consecutive patients. Neurosurgery 2011; 68: 108-116; discussion 116.

5) Yuta K, Yutaka M, Taichiro K, et al: Self-expanding stents placement for vertebral artery ostium stenosis: a report of two cases. NKC No Kekkannai Chiryo 2019; 4: 136-143. (in Japanese)

6) Takemoto K, Iwaasa M, Uda K, et al: A case of occlusion due to acute in-stent thrombosis after carotid artery stenting. Jpn J Neurosurg (Tokyo) 2009: 18: 305-311. (in Japanese)

7) Kornowski R, Hong MK, Tio FO, et al: In-stent restenosis: contributions of inflammatory reponses and arterial injury to neointimal hyperplasia. J Am Coll Cardiol 1988: 31: 224-230. 
8) Köster R, Vieluf D, Kiehn M, et al: Nickel and molybdenum contact allergies in patients with coronary in-stent restenosis. Lancet 2000; 356: 1895-1897.

9) Shah QA, Georgiadis AL, Suri MF, et al: Cutting balloon angioplasty for carotid in-stent restenosis: case reports and review of the literature. J Neuroimaging 2008; 18: 428-432.

10) Akiyama $Y$, Moritake K, Miyazaki T, et al: Cutting balloon angioplasty for carotid artery in-stent stenosis supported by three-dimensional rotational angiography with automated vessel analysis software. Neurol Med Chir (Tokyo) 2008; 48: 235-238; discussion 238.

11) Heck D: Results of cutting balloon angioplasty for carotid artery in-stent restenosis in six patients: description of the technique, long-term outcomes, and review of the literature. J Neurointerv Surg 2009; 1: 48-50.

12) Albiero R, Nishida T, Karvouni E, et al: Cutting balloon angioplasty for the treatment of in-stent restenosis. Catheter Cardiovasc Interv 2000; 50: 452-459.

13) Muramatsu T, Tsukahara R, Ho M, et al: Efficacy of cutting balloon angioplasty for in-stent restenosis: an intravascular ultrasound evaluation. J Invasive Cardiol 2001; 13: 439-444.

14) Albiero R, Silber S, Di Mario C, et al: Cutting balloon versus conventional balloon angioplasty for the treatment of in-stent restenosis: results of the restenosis cutting balloon evaluation trial (RESCUT). J Am Coll Cardiol 2004; 43: 943-949.

15) Park SJ, Kim KH, Oh IY, et al: Comparison of plain balloon and cutting balloon angioplasty for the treatment of restenosis with drug-eluting stents vs bare metal stents. Circ J 2010; 74: 1837-1845.

16) Qiu Z, Liu J, Huang R, et al: Incidence, risk, and treatment of binary restenosis after vertebral artery stenting. Catheter Cardiovasc Interv 2020: 96: 404-409. 